EDUCATION

Research, hnovation and Solutions on-line $\theta$
PSYCHOLOGY

HOD+i

\section{Electronic Journal of Research}

in Educational Psychology

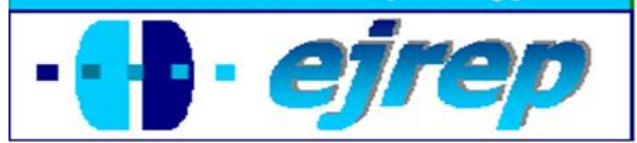

\title{
Educar en creatividad: un programa formativo para maestros de Educación Infantil basado en el juego libre
}

\section{Laura Jiménez y Ma Dolores Muñoz}

Departamento de Psicología, Facultad de Educación, Universidad de Castilla-La Mancha, Cuenca

\section{España}

Correspondencia: Laura Jiménez Márquez. Departamento de Psicología. Facultad de Educación. Campus Universitario s/n. 16071. Cuenca. España. E-mail: laura.jimenez@uclm.es 


\section{Resumen}

Introducción. El objetivo de esta investigación es, por un lado, conocer las concepciones incorrectas que los maestros en formación de Educación Infantil presentan sobre la educación de la creatividad y, por otro, desarrollar un programa formativo de tipo práctico, basado en técnicas de juego libre, que puedan aplicar en su futuro profesional.

Método. Para ello, 36 estudiantes de tercer curso de Grado de Maestro en Educación Infantil fueron evaluados, antes y después del entrenamiento, mediante una encuesta de 16 ítems que incluía conocimientos teóricos y aplicados sobre el proceso creativo.

Resultados. Los resultados evidenciaron un gran número de creencias incorrectas iniciales, sobre todo de tipo práctico, relacionadas con estrategias de diseño de la situación de juego libre para potenciar la creatividad, que fueron modificadas tras haber experimentado dicho programa formativo.

Discusión y Conclusión. Los datos enfatizan la necesidad de incorporar en el curriculum de los maestros un bagaje de conocimientos aplicados que les permitan educar correctamente la creatividad en la infancia.

Palabras Clave: educación en creatividad, formación de maestros, creencias falsas, juego infantil, escucha empática no participativa. 


\title{
Educating for creativity: A Preschool Teachers training course based on free play
}

\begin{abstract}
Introduction. The objective of this research is, firstly, to know the misconceptions that preservice Preschool teachers have about the education for creativity and, secondly, to develop a practical training course, based on free-play techniques, which they can apply in their professional future.
\end{abstract}

Method. To carry out that, 36 third-year Preschool teacher-students were evaluated before and after training, by theory and applied-based 16 test questions on the creative process, based on free-play, which they can apply in their professional future.

Results. Results showed a large number of incorrect beliefs, especially applied ones, regarding to the design of a free-play situation used as a resourse to enhance creativity, which were modified after experiencing the training course.

Discussion and Conclusion. The data emphasize the need to incorporate a wealth of applied knowledge in the teachers study program for promoting creativity in early childhood education.

Keywords: educating for creativity, teachers training education, incorrect beliefs, childhood play, non-participatory empatic listening

Received: 06/05/12 Initial acceptance: 07/08/12 Final acceptance: 11/13/12 


\section{Introducción}

Desde hace décadas, nuestra sociedad está demandando una mayor atención a la estimulación del desarrollo de la creatividad en el ámbito educativo (Arnheim, 1999; De Bono, 2011; De la Torre, 1982; Gardner, 1999; Guilford, 1991; Rogers, 1991). De hecho, la educación en creatividad puede ser un recurso valioso para mejorar la calidad de la enseñanza en todos los niveles del sistema educativo (De la Torre y Violant, 2006).

Tradicionalmente se consideró que la creatividad era una cualidad, o un don, que solamente poseían unos pocos. Hoy en día, sin embargo, está comprobado que la creatividad es una capacidad que todos poseemos en menor o mayor medida (de hecho se da más en niños normales que en niños prodigio) y, como cualquier capacidad, es susceptible de ser educada, lo que la convierte en una parte esencial del proceso de aprendizaje (De Bono, 2011; Del Moral Pérez, 1999; De la Torre, 1982; Gardner, 1999; López-Martínez, 2008; Lowenfeld y Brittain, 2008). El pensamiento creativo se caracteriza por ser fluido (i.e., cantidad de productos creativos que generamos), flexible (i.e., cantidad de productos diferentes que podemos crear), elaborado (i.e., detallado y específico) y original (i.e., novedoso) (Garaigordobil y Pérez, 2002; Prieto, López, Bermejo, Renzulli y Castejón, 2002). Su desarrollo depende de la estimulación de uno de los dos modos de funcionamiento que utiliza nuestra mente para procesar la información procedente de experiencias anteriores. Uno es reproductivo (vertical o lógico) y el otro creativo (lateral o divergente). Algunos autores llaman la atención sobre la necesidad de que la educación actual potencie más el desarrollo del pensamiento lateral y divergente, porque habitualmente suele ocuparse exclusivamente del vertical y lógico (De Bono, 2011; De la Torre y Violant, 2006).

La creatividad es igualmente una capacidad inherente e indisociable del desarrollo infantil. Precisamente, desde hace varias décadas, diversos autores sitúan los orígenes de la actividad creativa e imaginativa en las manifestaciones tempranas del juego del niño (p.e., Aucouturier, Darrault y Empinet, 1985; Lapierre y Lapierre, 1982; Vigotsky, 1982). Afirman además que, más adelante, sus producciones creativas van cambiando, de tal forma que cuando el niño domina suficientemente las habilidades grafomotoras se puede expresar también de forma original a través del dibujo y, posteriormente, cuando ha establecido el lenguaje como medio de comunicación, puede hacerlo a través de producciones literarias sencillas, orales o escritas (Muñoz, 2011; Vigotsky, 1982). 
Educar en creatividad es un objetivo explícito que forma parte de las finalidades de todos los sistemas educativos de los países desarrollados y de las apuestas de la UNESCO en materia de educación. En España se recoge igualmente en los proyectos educativos de centro, aunque esto puede implicar un riesgo. En la etapa de Educación Infantil, la manifestación de esta capacidad es altamente susceptible de ser estimulada, pero también podría llegar a ser peligrosamente inhibida o bloqueada (p.e., De la Torre; 1982; De la Torre y Violant, 2006; Franco Justo, 2008; Gardner, 1999; Lowenfeld y Brittain, 2008; Vigotsky, 1982). En este sentido, una de las mejores formas de potenciar la creatividad en la infancia, y que implica menos riesgos, es mediante el juego (p.e., Aucouturier et at., 1985; Gutiérrez Delgado, 2004; Howard-Jones, Taylor y Sutton, 2002; Lapierre, 1997; Saracho, 1992). Diversos expertos en juego y psicomotricidad defienden igualmente que la modalidad de juego libre sería la más adecuada, ya que al no necesitar la utilización del lenguaje oral, es más accesible para niños de cualquier edad, nivel intelectual, cultura o condición y crea un clima adecuado que facilita la emergencia del acto creativo de forma natural (Aucouturier et al., 1985; Lapierre y Lapierre, 1982).

Los programas que han analizado los efectos de introducir el juego educativo para fomentar el desarrollo han encontrado incrementos en la fluidez, flexibilidad, elaboración y originalidad de los alumnos, es decir, en su pensamiento creativo (Alonso y Aguirre, 2004; Chávez, Zacatelco, y Acle, 2009; Duarte, 2003; Garaigordobil, Maganto y Etxeberría, 1996; Prieto, López, Bermejo, Renzulli y Castejón, 2002). Algunos estudios longitudinales, como los realizados por Nickerson (1999) y Plucker y Renzulli (1999), han señalado, igualmente, que el entrenamiento para potenciar la creatividad durante el periodo de la Educación Infantil correlaciona con los niveles encontrados en esos mismos niños en etapas educativas superiores. Incluso, se ha mostrado que su entrenamiento produce efectos que pueden continuar hasta la vida adulta (De Bono, 2011; De la Torre y Violant, 2006; Kaufman y Beghetto, 2009; Lowenfeld y Brittain, 2008; Vigotsky, 1982).

En este punto, sin dejar al margen la importancia del juego educativo, no se puede olvidar que lo fundamental no es el juego en sí, sino la forma de jugar (Gutiérrez Delgado, 2004). Esto es, el juego debe ser creativo como fin en sí mismo y debe estar unido a la idea de libertad, lo que implica que las actividades educativas deben desarrollarse en ese marco (p.e, De la Torre y Violant, 2006; Del Moral, 1999; Lapierre y Lapierre, 1982; Lapierre, 1997; Rogers, 1991). En palabras de Trigo (2002), el juego educativo no debe ser excesiva- 
mente rígido ni prefijado porque no daría cabida a la imaginación. Por muy adecuado que resulte un juego, si coarta toda iniciativa, debido a que las reglas están claramente definidas, convertimos el juego en un mero instrumento. Esta premisa es fundamental puesto que el niño todavía no ha conseguido la madurez necesaria para oponerse eficaz y constructivamente a la presión dirigida y programada desde el exterior (García Ga Henche, 1981). Empíricamente, los estudios realizados por Cordazzo, Almeida y Vieira (2011) y González Moreno, Solovieva y Quintanar (2011), empleando un programa de entrenamiento en técnicas basadas en el juego libre, han confirmado recientemente que se ha estimulado el proceso de desarrollo motor, cognitivo y social de los niños que participaron.

A raíz de todo lo expuesto, resulta evidente que potenciar la creatividad durante la infancia en el ámbito educativo resulta fundamental. Pero, ¿cómo puede enfrentarse a ese reto el maestro de Educación Infantil?, ¿está preparado?, o mejor dicho, ¿recibe formación suficiente para poder hacerlo? El sistema educativo debe asumir el reto de crear contextos de aprendizaje que fomenten la creatividad en los niños, valorando la libre expresión de todos y cada uno de ellos, proporcionándoles oportunidades y momentos de expresión en un ambiente adecuado (De la Torre y Violant, 2006; Lowenfeld y Brittain, 2008; Rogers, 1991). Huelga decir que los maestros de Educación Infantil deberían tener una formación específica, tanto a nivel teórico como práctico, sobre la génesis de la creatividad en la infancia y los recursos y estrategias para estimularla. El análisis de los conocimientos teóricos que los maestros en formación tienen sobre la creatividad y el modo de fomentarla en el aula a través de recursos idóneos ha sido precisamente uno de los objetivos de este trabajo.

\section{Aspectos teóricos clave en la creatividad, según la Teoría de Vigotsky}

Para la delimitación del concepto de creatividad se ha partido de la teoría de Vigotsky (1982), puesto que se considera que los docentes actuales y en formación pueden seguir encontrando en sus postulados una valiosa información. En congruencia con los objetivos de este trabajo, los principios clave a la hora de que un profesor se encuentre preparado para implementar un programa formativo han sido los siguientes:

\section{1. y 2. El niño tiene más fantasía que el adulto, pero el adulto tiene más imaginación} que el niño. En palabras de Vigotsky, "la actividad creadora de la imaginación se encuentra en relación directa con la riqueza y la variedad de la experiencia acumulada por el hombre. 
Por eso, el niño tiene más fantasía que el adulto, pero la imaginación del niño es más pobre por ser menor su experiencia" (p. 17).

3. El acto creativo es fruto de más de una persona. Se puede encontrar creación siempre que el ser humano imagina, combina, modifica y crea algo nuevo, por insignificante que sea. Sin embargo, para Vigotsky lo creativo tiene siempre una connotación social y, por ello, defiende que no hay inventos (estrictamente) individuales porque en todo acto creativo siempre participan otras colaboraciones anónimas (p. 16).

4. La creatividad surge de la experiencia anterior. $\mathrm{Si}$, como se ha indicado, la imaginación depende de la experiencia acumulada, en palabras de Vigotsky "sería un milagro que la imaginación pudiese crear algo de la nada, o dispusiese de otras fuentes de conocimiento distintas de la experiencia pasada" (p. 16).

5. La creatividad es potenciada por las situaciones de necesidad. Para que se produzca la creación, es necesario que haya una necesidad. En la base de toda acción creadora reside siempre una inadaptación, anhelos, deseos, etcétera y, precisamente, son estas necesidades las que promueven el proceso imaginativo (p. 36).

6. La actividad creadora surge gradualmente. La actividad creadora no aparece repentinamente, sino con lentitud y gradualmente, ascendiendo desde formas elementales y simples a otras más complicadas (p. 15).

7. El niño es creativo desde su más tierna infancia. Los procesos creadores se advierten ya con todo su rigor desde la más tierna infancia y se observan, sobre todo, en los juegos infantiles. Cuando los niños juegan, no se limitan a recordar, sino que reelaboran y edifican nuevas realidades (p. 12).

8. Un buen educador que quiera potenciar la creatividad en el niño debe partir de dar libertad a la acción del niño. Vigotsky aconseja que el papel del educador es favorecer la libre expresión del niño para permitir la salida de su potencial creador (p. 53). El papel del educador consiste en potenciar la expresión libre en cualquiera de sus formatos, sea el juego, el dibujo o la creación literaria (p. 54).

\section{Potenciar la creatividad de forma aplicada, mediante el juego libre}

Como ya se ha defendido a lo largo de esta introducción, la escuela es el lugar en el que, de forma sistemática y planificada, se puede empezar a potenciar el desarrollo de la creatividad, al mismo tiempo que se vela por el desarrollo óptimo e integral del niño (Arnheim, 1999; De Bono, 2011; Del Pozo, 2006; Gardner, 1999; Lapierre y Lapierre, 1982; Vigotsky, 
1982). Y de todos los recursos educativos propios de la Educación Infantil, la actividad física, y más concretamente la psicomotricidad y el juego, pueden ser uno de los medios más adecuados para comenzar a potenciar estas habilidades (Garcés y Gómez, 2003; Muñoz, 2011; Viciana y Conde, 2002).

Siguiendo a Lapierre y Lapierre (1982), se ha empleado la técnica del juego libre, como un recurso propio de la psicomotricidad relacional, para potenciar la creatividad junto al desarrollo integral del niño de 0 a 6 años. Se ha seleccionado esta técnica porque, al ser "no directiva" y "no oral", utiliza la comunicación natural cuerpo a cuerpo -que es innata y universal-, y permite el entendimiento entre personas de cualquier edad, nivel intelectual y cultura. La comunicación interpersonal se produce "simplemente jugando en libertad unos junto a otros, sean quienes sean, y pase lo que pase" (p. 19).

Según la teoría de Lapierre y Lapierre (1982), Lapierre (1997) y Aucouturier et al. (1985), la actividad de juego no se produce siempre al mismo nivel, sino que evoluciona pasando consecutivamente por cuatro fases: (1) juego habitual (los objetos son lo que son, y se utilizan de forma habitual - p.e., una pelota se emplea para meter un gol en una portería-), (2) juego creativo (los objetos siguen siendo lo que son, pero se utilizan de una forma que no se han utilizado nunca -p.e., una pelota se emplea para limpiar el suelo de la portería-), (3) juego simbólico (el objeto además de lo que es, adquiere otro significado simbólico -siguiendo con el ejemplo anterior, la pelota se convierte en un perrito que vive en una casita, que es la portería-), y (4) juego fantasmático (dentro del juego simbólico alguien revive alguna experiencia real de su pasado, recuperando imágenes y emociones -p.e., la pelota que se ve como un perrito, le recuerda al niño a un perro real que tuvo y quería mucho-). Como se expondrá en el procedimiento empleado, se ha partido del juego habitual hasta llegar al juego creativo, intentando permanecer en este nivel sin evolucionar a las otras fases.

\section{Objetivos e hipótesis}

Este trabajo ha planteado un doble objetivo. En primer lugar, identificar las creencias, correctas e incorrectas, que los profesores de Educación Infantil en formación tienen sobre el proceso creativo y el rol que el maestro debe desempeñar para fomentarlo mediante el juego libre. Dicho análisis mostrará, por un lado, si existen (o no) deficiencias y, en caso afirmativo, si los tipos de creencias correctas o incorrectas se agrupan en mayor medida en torno a dichas 
propuestas teóricas o aplicadas. Desde nuestro punto de vista, y dado que la formación universitaria de los participantes ha sido de carácter eminentemente teórico, se considera que los maestros (en formación) presentarán un porcentaje de ideas incorrectas sobre la creatividad, y más aún sobre el papel que puede desempeñar el maestro para estimularla empleando el juego libre.

En segundo lugar, teniendo en cuenta las investigaciones que indican la importancia del juego educativo y su relación con la creatividad, se pretende crear un programa formativo de aprendizaje por descubrimiento en el que los futuros profesores puedan experimentar los aspectos técnicos para que los niños alcancen el nivel creativo del juego libre. Una vez concluido el entrenamiento se espera que se confirme también la hipótesis de que los conocimientos que habrán alcanzado los futuros maestros, a nivel teórico y sobre todo práctico, acerca de su papel en el desarrollo de la creatividad infantil, serán los adecuados para poder programar espacios en los que se propicie su estimulación mediante el juego.

\section{Método}

\section{Participantes}

En este estudio han participado 36 alumnos egresados en el tercer curso de grado de Maestro en Educación Infantil de la Facultad de Educación de Cuenca (Universidad de Castilla-La Mancha). Fueron seleccionados todos los estudiantes que estaban cursando la mención cualificadora en Lenguajes creativos.

\section{Material y procedimiento}

La evaluación se produjo en dos momentos diferentes. En primer lugar, antes de tener ningún tipo de formación previa sobre el juego, a los alumnos se les administraba una encuesta formada por 16 preguntas de dos alternativas, una correcta y otra incorrecta, que tenía como objetivo explorar los juicios previos de los profesores en torno al proceso creativo y al rol que cumple el maestro para potenciarlo mediante el juego libre (ver Tabla 1). Dicha encuesta fue diseñada ad-hoc por los investigadores. La mitad de las preguntas que componían el cuestionario estaban basadas en las propuestas teóricas de Vigotsky (1982) y la otra mitad versaban sobre las propuestas de Lapierre y Lapiere (1982) para practicar el juego libre con niños de 
Educación Infantil. El orden de las preguntas fue contrabalanceado de forma aleatoria en el cuestionario real.

Tabla 1. Cuestionario empleado para evaluar las creencias de los profesores

\begin{tabular}{|c|c|c|c|}
\hline \multicolumn{2}{|c|}{ Preguntas teóricas (basadas en Vigotsky) } & \multirow[t]{2}{*}{$(\mathrm{A})$} & \multirow[t]{2}{*}{ (B) } \\
\hline 1. Imaginación & $\begin{array}{l}\text { ¿Quién tiene más imaginación? } \\
\text { El niño (A) / o el adulto (B) }\end{array}$ & & \\
\hline 2. Fantasía & $\begin{array}{l}\text { ¿Quién tiene más fantasía? } \\
\text { el niño (A) / o el adulto (B) }\end{array}$ & & \\
\hline 3. Creador & $\begin{array}{l}\text { ¿Todo acto creativo es fruto de: } \\
\text { una sola persona }(\mathrm{A}) / \text { o de más personas }(\mathrm{B})\end{array}$ & & \\
\hline 4. Origen & $\begin{array}{l}\text { ¿De dónde surge la creatividad? } \\
\text { De la nada (A) / De la experiencia (B) }\end{array}$ & & \\
\hline 5. Motivo & $\begin{array}{l}\text { ¿Qué favorece más la creatividad? } \\
\text { Tener necesidades (A) / No tenerlas (B) }\end{array}$ & & \\
\hline 6. Proceso & $\begin{array}{l}\text { ¿La actividad creadora en cada persona surge?: } \\
\text { De repente (A) / Gradualmente (B) }\end{array}$ & & \\
\hline 7. Etapa & $\begin{array}{l}\text { ¿Puede ser creativo un niño desde su más tierna infancia? } \\
\text { Sí (A) / No, porque necesita alcanzar cierta madurez (B) }\end{array}$ & & \\
\hline 8. Libertad & $\begin{array}{l}\text { Un buen educador que quiera potenciar la creatividad en el } \\
\text { niño ¿de qué premisa debe partir? Dar libertad a la acción } \\
\text { del niño (A) / Programar un entrenamiento previo (B) }\end{array}$ & & \\
\hline Preguntas prácti & as (basadas en aplicación de Lapierre) & (A) & (B) \\
\hline 1. Entorno & $\begin{array}{l}\text { ¿Qué favorece más la creatividad? Usar: Pocos (A) / Mu- } \\
\text { chos materiales (B) }\end{array}$ & & \\
\hline 2. Instrucciones & $\begin{array}{l}\text { ¿Cómo potenciarías la creatividad del niño en el juego? } \\
\text { Dándole instrucciones (A) / No diciéndole nada (B) }\end{array}$ & & \\
\hline 3. Materiales & $\begin{array}{l}\text { ¿Es mejor que...?: El niño elija los materiales (A) } \\
\text { / Que los elija el adulto (B) }\end{array}$ & & \\
\hline 4. Mirada & $\begin{array}{l}\text { ¿Cómo afecta la mirada del adulto en el proceso creativo } \\
\text { del niño? Lo estimula (A) / Lo bloquea (B) }\end{array}$ & & \\
\hline 5. Lenguaje & $\begin{array}{l}\text { ¿Cómo afectan las palabras del adulto en el proceso creati- } \\
\text { vo del niño? Lo estimula (A) / Lo bloquea (B) }\end{array}$ & & \\
\hline 6. Empatía & $\begin{array}{l}\text { ¿Se puede "estar presente en la actividad del niño" y com- } \\
\text { prenderle, sin que el profesor mire al niño y sin que le } \\
\text { hable? Si (A) / No (B) }\end{array}$ & & \\
\hline $\begin{array}{l}\text { 7. Tipo de jue- } \\
\text { go }\end{array}$ & $\begin{array}{l}\text { ¿Qué tipo de juego es más eficaz para estimular el desarro- } \\
\text { llo de la creatividad en educación infantil? } \\
\text { El juego dirigido, (A) / El juego libre, (B) }\end{array}$ & & \\
\hline $\begin{array}{l}\text { 8. Espacio / } \\
\text { postura }\end{array}$ & $\begin{array}{l}\text { ¿Tiene importancia la distancia espacial del cuerpo del } \\
\text { adulto y su postura, respecto a la actividad creativa del } \\
\text { niño? No (A) / Si (B) }\end{array}$ & & \\
\hline
\end{tabular}


En segundo lugar, durante un periodo temporal de dos meses, los alumnos participaron en un programa de entrenamiento compuesto por un total de nueve sesiones prácticas de tres horas de duración, una por semana, en las que tuvieron la ocasión de experimentar el método de juego libre en dos modalidades, desde el punto de vista del niño y desde el punto de vista del maestro empático no-participativo y no-directivo. Los estudiantes se enfrentaban a las prácticas sin haber recibido ninguna formación específica previa, de forma que pudieran extraer por ellos mismos evidencias sobre la influencia que el tipo de juego ejerce en el desarrollo de la creatividad. Las sesiones de juego libre se distribuyeron como sigue:

\section{Juego libre. Rol de alumno. Sesiones 1-6}

Como ya se ha indicado en la introducción de este trabajo, el método de juego libre basado en la metodología de Lapierre y Lapierre (1982) y Lapierre (1997) es una modalidad desconocida para los profesores en formación, que requiere un desarrollo en el tiempo a medio plazo para poder llegar a experimentar una evolución espontánea en los cuatro niveles: (1) juego habitual, (2) juego creativo, (3) juego simbólico, y (4) juego fantasmático.

En concreto, se experimentaron los dos primeros niveles de juego, el habitual y el creativo, siguiendo las recomendaciones de los autores (Lapierre y Lapierre, 1982, pp. 27-49). El nivel de juego creativo surge desde el juego habitual, pero no se mantiene estable, sino que sigue evolucionando de forma imprevista en cada sujeto hasta llegar al nivel de juego simbólico y fantasmático (Lapierre, 1997, p. 171). Por ello, conforme a los objetivos de esta investigación, se modificó una de las variables. Esta adaptación consistió en aislar la variable inductora del cambio de juego creativo a los otros dos niveles, que es fundamentalmente la participación del "cuerpo del adulto". Utilizando la técnica ortodoxa, el cuerpo del adulto está disponible para el niño y es un objeto más con el que puede jugar cuando quiera (p. 71). Puesto que en esta investigación se pretendía que los participantes se mantuviesen en el nivel de juego creativo, se incorporó la norma de que el cuerpo del maestro no pudiese estar disponible como objeto de juego. De esta manera, la postura del adulto no era de "igual a igual". El maestro estaba presente pero su relación era de escucha empática no participativa, esto es, no perturbaba ni interfería en la expresión libre del niño. A este tipo de relación, basada en los postulados de Rogers (1991) y consistente en "estar centrados en el otro", la hemos denominado "escucha empática no participativa". 
En esta primera fase, de seis sesiones prácticas, todos los participantes desempeñaban el rol de niños. La única consigna oral que se les facilitaba era una invitación a entrar en la situación: “¿A qué se puede jugar con...?” (p.e., pelotas, aros, cuerdas, telas, voz sin lenguaje, nada, etc., en función del objeto que se seleccionase cada día). La premisa que guiaba el juego era "que fuesen libres para hacer (o no hacer) lo que quisiesen desde ese momento, con la única prohibición de emplear el lenguaje oral". Por tanto, la actividad de las sesiones se desarrollaba según el deseo de los participantes, pero sin lenguaje oral (ver programa de entrenamiento y evolución de los participantes en el Anexo).

La duración de la fase de juego solía oscilar entre una hora y una hora y media. Una vez finalizado el juego, cuando la mayoría de los participantes tomaban posturas de descanso en el suelo, se entraba en un estado de relajación libre que duraba de 10 a 15 minutos aproximadamente. El final de la práctica se cerraba con una verbalización colectiva en la que los propios estudiantes expresaban sus descubrimientos. Es decir, el aprendizaje vivido se plasmaba en contenidos teóricos, conceptualizando sus propias vivencias, pero sin analizar las teorías de otros autores.

El transcurso entre las tres actividades (p.e., juego, relajación y verbalizaciones) se acompañaba mediante el cambio de la música de fondo. De esta forma, y en todo momento, el investigador representaba un papel meramente observador, no directivo y sin mediación del lenguaje oral para que se produjese y mantuviese el clima de juego libre, con comunicación empática no participativa y no oral con el grupo.

\section{Juego libre. Rol de maestro empático. Sesiones 7-9}

En las últimas 3 sesiones de juego libre se cambió la metodología. Se crearon tres grupos de alumnos y se les solicitó que uno de ellos asumiese el rol de maestro empático en cada sesión. Los dos subgrupos restantes actuaban según las consignas y premisas de las primeras sesiones de entrenamiento en juego libre. Estas sesiones tenían la función de permitir a los profesores en formación experimentar el juego libre también desde el lado del maestro (ver programa de entrenamiento y evolución de los participantes en el Anexo).

Los participantes que intervenían como maestros en las sesiones recibían la consigna de actuar de forma empática no participativa. Para controlar la aplicación de esta variable, 
todos los adultos que desempeñaban el rol de maestros debían observarse entre sí y comprobar que cada uno de ellos cumplía las normas y no interfería en el desarrollo del juego libre del resto del grupo que seguían actuando como alumnos, esto es, manteniéndose como un "observador empático" y no como un "director de escena", un "igual con el niño", un "consejero", un "evaluador" o "un objeto". Si su presencia dejaba de ser "observación empática no participativa" se analizaban posteriormente con el grupo las consecuencias que hubiese provocado dichas interferencias en el juego.

En síntesis, las normas de participación del adulto para intervenir apoyando -y no inhibiendo- la actividad creativa de los niños, según nuestra propuesta, consistían en: (1) estar físicamente "presente y abierto a la comunicación”, (2) cognitivamente "descentrados", “centrados en el otro", y "en la escucha del niño siguiendo la evolución de sus producciones sin emitir mensajes", (3) emocionalmente "estable" y en un estado permanente de "estar bien" independientemente de lo que hiciese cada niño, y (4) socialmente "no directivo". Mantenerse en este estado puede parecer fácil porque aparentemente es "no hacer nada", pero lo habitual suele ser comportarse mecánicamente de forma totalmente opuesta. De ahí, la necesidad del entrenamiento.

Para evaluar las creencias y actitudes de los estudiantes dos meses después tras la aplicación del programa, los participantes volvieron a cumplimentar la encuesta destinada a evaluar si se había producido el cambio de creencias sobre el proceso creativo y el rol del maestro en el juego libre.

Por último, ya que el entrenamiento estuvo a cargo del equipo investigador, para que los investigadores fuesen ciegos a los resultados, el análisis de los cuestionarios previos no se realizó hasta que no finalizaron las sesiones prácticas.

\section{Diseño y análisis de datos}

Se empleó un estudio transversal de tipo cuasi-experimental en el que se se comparó un grupo en la medición pre-tratamiento y la medición post-tratamiento. Para comprobar la existencia de diferencias estadísticamente significativas, se realizó un ANOVA mixto de dos factores con dos niveles de análisis, esto es, dos Momentos de Medición (Pre vs. Post- 
entrenamiento) y dos Tipos de contenido (Teórico vs. Práctico) con medidas repetidas en ambos factores.

\section{Resultados}

El ANOVA mixto 2 (Momento de Medición: Pre-entrenamiento vs. Postentrenamiento) * 2 (Tipo de contenido: Teórico vs. Aplicado) con medidas repetidas en ambos factores indicó que fueron significativos los efectos principales del factor Momento de Medición $\left(\mathrm{F}(1,35)=308.59, \mathrm{p}<.01, \eta \mathrm{p}^{2}=.9\right)$ y la interacción entre ambos factores $(\mathrm{F}(1,35)$ $=42.55, \mathrm{p}<.01, \eta \mathrm{p} 2=.55$ ), pero no el Tipo de contenido (ver Tabla 2 ).

Tabla 2. Medias y Desviaciones Típicas (entre paréntesis) de las respuestas correctas

\begin{tabular}{lcc} 
& Pre-entrenamiento & Post-entrenamiento \\
\hline Ítems teóricos basados en la teoría & 3.33 & 6.47 \\
de Vigotsky & $(1.35)$ & $(1.21)$ \\
\hline Ítems aplicados basados en la & 2.31 & 7.67 \\
propuesta de Lapierre & $(1.51)$ & $(0.53)$ \\
\hline
\end{tabular}

Nota: La puntuación máxima es de 8 puntos

Por lo que respecta al Momento de Medición, conforme a lo esperado, los participantes experimentaron una gran mejora en la evaluación Post-entrenamiento $(\mathrm{M}=7.07, \mathrm{SD}=.87)$ en comparación con la medición Pre-entrenamiento $(\mathrm{M}=2.82, \mathrm{SD}=1.43)$. En primer lugar, cabe destacar que la evaluación previa mostró que, de forma global, tan sólo el $35.2 \%$ de las creencias sobre la creatividad incluidas en el cuestionario eran identificadas correctamente por los alumnos. Sin perder de vista que los participantes de este estudio habían superado el ecuador de los estudios de grado de Maestro en Educación Infantil, es evidente que continuaban permaneciendo una serie de concepciones incorrectas, de tipo teórico y práctico, en relación con la génesis de la creatividad en la infancia y la forma de favorecerla mediante el juego.

En concreto, esto se ha visto plasmado en el análisis del número de preguntas que los alumnos fueron capaces de contestar correctamente. Así, la mitad de los participantes mostraba un conocimiento bajo o muy bajo, reconociendo un total que oscilaba entre 1 y 5 alternativas correctas de las 16 preguntas que se incluían en el cuestionario, mientras que la otra mitad se situaba en un nivel medio o medio-bajo, identificando entre 6 y 9 afirmaciones correctas. 
En segundo lugar, como ya se ha comentado, dos meses después de haber finalizado el entrenamiento basado en el aprendizaje por descubrimiento de los contenidos, los alumnos eran capaces de identificar, como media, un $88.4 \%$ de las afirmaciones correctas. De nuevo, analizando el porcentaje de alumnos y el número de preguntas que respondieron correctamente tras el entrenamiento, ningún alumno identificó menos de 11 respuestas correctas, ya que la amplia mayoría se situaba entre los 13 y 16 ítems (88.9\%) y el $16.7 \%$ alcanzaba la puntuación máxima. Estos resultados confirman que los estudiantes cambiaron significativamente de creencias empleando para ello su propia experiencia, lo que muestra, a su vez, la idoneidad del recurso formativo. En este punto cabe preguntarse, ¿cuáles fueron los juicios previos de los profesores en formación tenían más arraigados sobre el proceso creativo y el rol del maestro? Es decir, ¿qué tipo de creencias correctas estaban previamente asentadas y cuáles no? (ver tabla 3 ).

Tabla 3. Porcentaje de alumnos que responden adecuadamente a los ítems del cuestionario

\begin{tabular}{|c|c|c|c|}
\hline & & Pre-tratamiento & Post-tratamiento \\
\hline \multirow{8}{*}{$\begin{array}{l}\text { Preguntas teóricas } \\
\text { (basadas en Vigotsky) }\end{array}$} & 1. Imaginación & 8.3 & 75 \\
\hline & 2. Fantasía & 83.3 & 100 \\
\hline & 3. Creador & 33.3 & 61.1 \\
\hline & 4. Origen & 8.3 & 72.2 \\
\hline & 5. Motivo & 55.6 & 86.1 \\
\hline & 6. Proceso & 33.3 & 75 \\
\hline & 7. Etapa & 63.9 & 77.8 \\
\hline & 8. Libertad & 47.2 & 100 \\
\hline \multirow{8}{*}{$\begin{array}{l}\text { Preguntas prácticas } \\
\text { (basadas en Lapierre) }\end{array}$} & 1. Entorno & 11.1 & 88.9 \\
\hline & 2. Instrucciones & 13.9 & 97.2 \\
\hline & 3. Materiales & 13.9 & 83.3 \\
\hline & 4. Mirada & 50 & 100 \\
\hline & 5. Lenguaje & 25 & 100 \\
\hline & 6. Empatía & 30.6 & 100 \\
\hline & 7. Tipo de juego & 52.8 & 100 \\
\hline & 8. Espacio/postura & 33.3 & 97.2 \\
\hline
\end{tabular}


Las creencias teóricas incorrectas que los estudiantes presentaban en mayor medida, siguiendo la teoría de Vigotsky, eran que: "el niño tiene más imaginación que el adulto" y "la creatividad surge de la nada" (91.7\%). Sin embargo, tras las sesiones de entrenamiento, los estudiantes verbalizaban lo contrario, después de reflexionar sobre el hecho de que los niños no han tenido tantas experiencias con materiales diversos (como pelotas, aros, cuerdas, telas, entre otros), por lo que era menos probable que imaginasen combinaciones nuevas relacionando esos elementos. Igualmente, en el segundo caso, los alumnos partían de la intuición de que "crear" proviene de la nada, y después de la experimentación comprendían que el acto creativo había estado basado siempre en experiencias previas. De hecho, generalizando, no se encontró ningún ejemplo en el que la creación hubiese surgido de la nada.

Adicionalmente, en torno al $70 \%$ de los participantes consideraba también erróneamente que: "el acto creativo surge repentinamente" y que "es fruto de una única persona". En estos casos, los alumnos partían previamente de la concepción de que la creación surgía por "arte de magia". Pero cuando analizaban sus vivencias verificaban que el acto creativo tenía una fase de incubación que, en su caso, había pasado por una etapa anterior de juego habitual. La necesidad de dejar de jugar al mismo juego "siempre igual" había provocado la transformación: inadaptación-necesidad-deseo-creación, defendida por Vigostky (1982).

Centrándonos ahora en las creencias asociadas a la función que cumple el juego en el desarrollo de la creatividad, más del 85\% de los alumnos también erraban suponiendo que es mejor que el niño: "tenga muchos materiales", "pueda elegirlos" y "es mejor que las instrucciones sobre el juego provengan del adulto". Además, alrededor del 70\%, aproximadamente, fallaban igualmente al creer que: "las palabras del adulto estimulan al niño", "no se puede estar presente en el juego del niño sin mirarle ni hablarle" y, por último, "la distancia espacial del cuerpo del adulto y su postura no es importante”. Sin embargo, a raíz de su experiencia desempeñando tanto el rol de niños como el rol de adulto pudieron experimentar las consecuencias negativas de estas acciones en el grupo. Así, desde el rol de niños podían identificar qué conductas del maestro promovían su actividad creadora y cuáles las bloqueaban, inhibían o interrumpían -como quedaba patente en las verbalizaciones después de las sesiones-. Desde el rol del maestro, veían las dificultades que suponía mantenerse estables como maestros empáticos y la necesidad de recibir retro-alimentación de sus compañeros para saber, desde el punto de vista de múltiples observadores externos, la adecuación de sus actos y sus consecuencias. 
Después del entrenamiento, como se puede observar en la Tabla 3, en torno un porcentaje que oscila entre el 75 y el 100\% de las creencias evaluadas fueron detectadas de forma correcta, independientemente de que el conocimiento previo de los estudiantes fuese inadecuado, como es el caso de las afirmaciones señaladas anteriormente, o tuviesen conocimientos previos, como sucedía con la percepción inicial correcta de que: "el niño puede ser creativo desde la más tierna infancia" y "el niño tiene más fantasía que el adulto". De todos ellos, se debe destacar que gran parte de los contenidos de tipo práctico fueron identificados correctamente por el $100 \%$ de los participantes, en concreto los relativos a la técnica de escucha empática no participativa, y el papel que juega la presencia del adulto, sus palabras o su mirada. En la evaluación final los estudiantes tenían evidencias claras de que el juego es más creativo cuando: el maestro elige los materiales previamente, hay poca variedad de objetos, no se dan instrucciones y no se interfiere ni mediante la mirada, el lenguaje o incorporándose al juego. Es decir, cuando el maestro simplemente estaba presente con lo que se ha denominado "escucha empática no participativa".

Por otro lado, centrándonos ahora en los procesos que forman parte del pensamiento creativo, se observó igualmente que el juego de los alumnos en las últimas sesiones fue más: fluido (aumentando la cantidad de juegos y la velocidad de aparición), flexible (puesto que la gama de posibilidades de juegos dejó de limitarse a situaciones determinadas), elaborado (utilizando elementos más sutiles y complejos) y original (generando juegos inusuales, nunca vividos), tal y como se había hallado en estudios previos centrados en el juego educativo (Alonso y Aguirre, 2004; Chávez Soto et al., 2009; Duarte, 2003; Garaigordobil et al., 1996; Prieto et al., 2002).

Por el contrario, debe también señalarse que la creencia que quedó finalmente menos asentada fue si: "el acto creativo es fruto de una sola persona o de más". Si bien se les preguntó posteriormente a los estudiantes, por escrito, y afirmaron que consideraban que el acto creativo también se puede producir colectivamente con aportaciones consecutivas de varias personas, mantuvieron su idea inicial de que el acto creativo puede ser también una creación individual. No obstante, en palabras de Vigotsky, detrás de cada creación hay otro creador anónimo, por lo que nadie crea nada solo (p.e., un niño se puede inventar un juego nuevo con una pelota, pero ¿quién inventó la pelota?). 
Para finalizar, en lo que concierte a la interacción entre el Momento de Medición y el Tipo de contenido, tal y como se esperaba, los alumnos partían de un mayor conocimiento Teórico que Aplicado (i.e., $41.7 \%$ vs. $28.8 \%$ ) pero, al finalizar el entrenamiento que, por su naturaleza, tenía un carácter eminentemente práctico, el nivel final de conocimiento Aplicado fue superior que el Teórico (i.e., 80.9\% vs. 95.8\%, respectivamente) (ver Tabla 2).

En este punto, si queremos evaluar realmente el impacto del programa formativo, se debe tener en cuenta que, efectivamente, los alumnos partían de diferentes grados de conocimiento. Esto es, si bien es cierto que al finalizar el entrenamiento el nivel de éxito final era muy elevado, incluso llegando a alcanzar un $100 \%$ de éxito en algunos ítems, es igualmente cierto que en algunos contenidos el grado de cambio ha sido mayor.

Para conocer dicho impacto, se ha analizado el cambio producido en los contenidos Teóricos y Aplicados -en el estado previo y el final- en cada uno de los ítems que lo componían. Así, los resultados muestran que, a nivel Teórico, un $46.9 \%$ de las ideas incorrectas fueron modificadas. Previamente, los alumnos identificaban correctamente un 34\% de los principios teóricos, y dichos conocimientos se mantuvieron al final del entrenamiento, lo que indica eran conocidos por los alumnos y se vieron corroborados. También debe señalarse que tan solo un $11.5 \%$ de los principios teóricos que no estaban previamente asentados, no fueron modificados, es decir, no se vieron beneficiados por el programa formativo. A nivel Aplicado el cambio fue más espectacular. Como ya se ha indicado, los alumnos poseían menos conocimientos previos correctos, por lo que solo el $27.4 \%$ de las afirmaciones de tipo práctico eran señaladas correctamente, manteniéndose hasta el final del entrenamiento. En este caso, tan solo un 2.8\% de las concepciones incorrectas no se modificaron. Esto indicaría que el entrenamiento fue eficaz y produjo un cambio real en el nivel de conocimiento de los alumnos en el $68.4 \%$ de los casos.

A modo de recapitulación, estos resultados indican que las técnicas de entrenamiento de maestros en juego libre han sido adecuadas, tanto como recurso para cambiar las creencias de los profesores en formación sobre creatividad, como sobre la aportación de estrategias nuevas para fomentarla, en este caso, mediante el juego libre. Los datos finales muestran que, independientemente de que el grado de conocimiento inicial fuese mayor o menor, finalmente casi el $90 \%$ de las afirmaciones fueron reconocidas correctamente, lo cual estaría demostrando el éxito del programa formativo. El impacto de este aprendizaje por descubrimiento en su 
futuro profesional se espera que sea duradero, puesto que está basado en la certeza por experiencia, y no en contenidos teóricos que pueden llegar a percibirse como una posibilidad incierta.

\section{Conclusiones}

Tal y como se señalaba en la introducción de este trabajo, si la educación en creatividad es fundamental en el periodo de los 0 a 6 años, los formadores de los futuros maestros de Educación Infantil tenemos que asumir la responsabilidad de prepararles específicamente para que puedan adquirir esas competencias. El reto de educar en creatividad se contempla en los proyectos educativos de los centros, pero la mayoría de los profesores no sabe hacerlo de forma adecuada ni cómo llevarlo a la práctica sistemáticamente.

Para verificar esta carencia a nivel de formación de profesorado, uno de los objetivos previos fundamentales era comprobar las creencias de tipo teórico y aplicado que están interiorizadas en los futuros maestros, antes de comenzar un programa docente práctico. Efectivamente, se ha constatado que presentan una serie de creencias falsas, especialmente a nivel aplicado, que pueden hacer que, en su futura profesión docente, lejos de estimular la creatividad del niño, la inhiban o, incluso, la bloqueen.

No obstante, los resultados del entrenamiento han mostrado que la práctica en técnicas para fomentar el desarrollo de la creatividad, como el juego libre -con la incorporación del control de la no intervención del cuerpo del adulto como un elemento más del juego- han resultado eficaces y, por tanto, recomendables en la modificación de dichas concepciones respecto a las estrategias de intervención que debe adoptar el maestro en la escuela. Es cierto también que el impacto en el cambio de algunas concepciones teóricas erróneas, especialmente en la consideración de que "el acto creativo es fruto de una persona", ha sido menor. Al acceder los investigadores al resultado de las encuestas una vez había finalizado el programa, esta carencia se descubrió tarde. Desde nuestro punto de vista, y especialmente en los contenidos más teóricos, en futuros estudios sería recomendable que los alumnos tuviesen la ocasión de verbalizar sus experiencias, no solo en torno a los aspectos que ellos mismos destacaban libremente de la sesión, sino en relación a un conjunto de preguntas de profundización previamente diseñadas. 
La conclusión más importante que se desprende de este estudio es la necesidad de que la formación inicial de los maestros incluya contenidos teóricos sobre la génesis de la creatividad, fundamentados en programas eminentemente prácticos, basados en aprendizaje experimental. Estas experiencias garantizan: (1) que los profesores han vivido en primera persona como la manifestación de la creatividad se ve en ocasiones bloqueada o inhibida y, en otras, potenciada; y (2) que serán capaces en su futuro profesional de crear contextos educativos similares en los que los niños puedan expresar su potencial creador sin ningún tipo de bloqueo. En última instancia, si uno de los objetivos del sistema educativo es fomentar la creatividad infantil, incorporar en la educación de los profesores un bagaje experimental de conocimientos teóricos y aplicados, es un paso imprescindible y una garantía para conseguirlo.

No podemos finalizar sin poner de manifiesto que las limitaciones que pueden encontrar los maestros que deseen recorrer este camino son numerosas. En primer lugar, la falta de recursos adecuados para formar inicialmente al profesorado universitario encargado de entrenar a los futuros maestros. En segundo lugar, la rigidez de todo el sistema educativo y su resistencia a dar libertad a los niños. Por último, la filosofía de vida actual que potencia más las relaciones de los niños con los aparatos que con las personas. En definitiva, es un reto difícil, pero posible. 


\section{Referencias}

Alonso, L. y Aguirre, R. R. (2004). La escritura creativa en la escuela. Una experiencia pedagógica (de; con) juegos lingüísticos y metáforas. Revista de Pedagogía, 74 (XXV), 375-399.

Arnheim, R. (1999). Consideraciones sobre la educación artística. Barcelona: Paidós.

Aucouturier, B., Darrault, I. y Empinet, J. L. (1985). La práctica psicomotriz. Reeducación y terapia. Barcelona: Científico-Médica. .

Chávez, B.I., Zacatelco, F. y Aclei, G. (2009). Programa de enriquecimiento de la creatividad para alumnas sobresalientes de zonas marginadas. Electronic Journal of Research in Educational Psychology, 7(2), 849-876.

Cordazzo, S. T. D, Almeida, A. M. T. y Vieria, M. L. (2011). The influence of play in motor, cognitive and social. Educational Research, 2(9), 1472-1480.

De Bono, E. (2011). El pensamiento lateral. Manual de creatividad. Madrid: Paidós Plural (1 ${ }^{\text {a }}$ Ed. castellano 1991).

De la Torre, S. (1982). Educar en creatividad. Madrid: Editorial Narcea.

De la Torre, S. y Violant, V. (2006). Comprender y Evaluar la Creatividad. Un recurso para mejorar la calidad de la enseñanza (Vol. I). Málaga: Aljibe.

Del Moral, M. E. (1999). Tecnologías de la Información y la Comunicación (TIC). Creatividad y educación. Educar, 25, 33-52.

Duarte, B. E. (2003). Creatividad como un recurso psicológico para niños con necesidades educativas especiales. SAPIENS Universidad Pedagógica Experimental Libertador, 4(002), 1-17.

Franco, C. (2008). Creative relaxation, motor creativity, self-concept in a sample of children from Early Childhood Education. Electronic Journal of Research in Educational Psychology, 6(1), 29-50.

Garaigordobil, M. y Pérez, J. I. (2002). Efectos de la participación en el programa de arte Ikertze sobre la creatividad verbal y gráfica. Anales de psicología, 18(1), 95-110.

Garaigordobil, M., Maganto, C., y Etxeberría, J. (1996). Effects of a cooperative game program on socio-affective relationships and group cooperation capacity. European Journal of Psychological Assessment, 12(2), 140-151.

Garcés, E. J. y Gómez, A. (2003). Promoción de la actividad física y el deporte en niños y adolescentes. Manual de Psicología de la salud con niños, adolescentes y familias. Madrid: Pirámide. 
García Ga Henche, F. (1981). Creatividad e imagen en los niños. Madrid: M.E.C.

Gardner, H. (1999). Educación artística y desarrollo humano. Barcelona: Paidós Educador. $\left(1^{\mathrm{a}}\right.$ ed. castellano, 1994)

Guilford, J. (1991). Creatividad y Educación. Barcelona: Editorial Paidós.

González Moreno, C. X., Solovieva, Y. y Quintanar, R. L. (2011). Actividad reflexiva en preescolares: perspectivas psicológicas y educativas. Universitas Psychologica, 10(2), 423-440.

Gutiérrez Delgado, M. (2007). La bondad del juego, pero... EA, Escuela abierta: revista de Investigación Educativa, 7, 153-182.

Howard-Jones, P., Taylor, J. y Sutton, L. (2002). They effect of play on the creativity of young children during subsequien activity. Early Child Development and Care, 172(4), 323-328.

Kaufman, J. C. y Beghetto, R. A. (2009). Beyond Big and Little: The Four C Model of Creativity. Review of General Psychology, 13(1), 1-12.

Lapierre, A. (1997). Psicoanálisis y Análisis Corporal de la Relación. Bilbao: Desclée de Brouwer.

Lapierre, A. y Lapierre, A. (1982). El adulto frente al niño de 0 a 3 años. Barcelona: Editorial Científico-Médica.

López, O. (2008). Enseñar creatividad. El espacio educativo. Cuadernos FHyCS-UNJu, 35, $31-40$.

Lowenfeld, V. y Brittain, L. W. (2008). Desarrollo de la capacidad intelectual y creativa. Madrid: Síntesis (traducción de la $1^{\text {a }}$ ed. 1987).

Muñoz, M. D. (2011). Desarrollo motor, trastornos y hábitos saludables. En J. J. Ricarte (Coord), Psicología de la salud infantil (pp. 133-170). Pamplona: Eunate.

Nickerson, R. S. (1999). Enhancing creativity. En R. J. Sternberg (Ed.), Handbook of creativity (pp. 392-430). New York, N.Y.: Cambridge University Press.

Plucker, J., y Renzulli, J. S. (1999). Psychometric approaches to the study of human creativity. En R. J. Sternberg (Ed.), Handbook of creativity (pp. 35-60). New York: Cambridge University Press.

Prieto, D., López, O., Bermejo, M., Renzulli, J. y Castejón, J. (2002). Evaluación de un programa de desarrollo de la creatividad. Psicothema, 14(2), 410-414.

Rogers, C. (1991). Libertad y Creatividad en la Educación. Barcelona: Editorial Paidós.

Saracho, O. N. (1992). Preschool children's cognifive style and implications for creativity. Creativity Research Journal, 5(1), 35-47. 
Trigo, E. (2002). Juegos motores y creatividad ( $3^{\mathrm{a}}$ ed.). Barcelona: Paidotribo.

Viciana, V. y Conde, J. L. (2002). El juego en el currículo de educación infantil. En J. A. Moreno (Coord), Aprendizaje a través del juego (pp. 67-97). Málaga: Aljibe.

Vigotsky, L. V. (1982). La creatividad y el arte en la infancia. Madrid: Akal. 
Anexo. Programa de entrenamiento de profesorado de Educación Infantil en técnicas de juego libre para favorece la creatividad

\begin{tabular}{|c|c|c|c|c|c|c|c|}
\hline ITEMS & SESIÓN I & SESIÓN II & SESIÓN III & SESIÓN IV & SESIÓN V & SESIÓN VI & $\begin{array}{l}\text { SESIONES } \\
\text { VII-IX }\end{array}$ \\
\hline Material & $\begin{array}{ll}\text { Pelotas } & \text { va- } \\
\text { riadas. }\end{array}$ & $\begin{array}{ll}\text { Aros } & \text { varia- } \\
\text { dos. } & \end{array}$ & $\begin{array}{ll}\text { Cuerdas } & \mathrm{y} \\
\text { telas. } & \end{array}$ & $\begin{array}{ll}\begin{array}{ll}\text { Todos } & \text { los } \\
\text { objetos } & \text { del } \\
\text { almacén. }\end{array}\end{array}$ & $\begin{array}{l}\text { Sin objetos, } \\
\text { sOlo con el } \\
\text { cuerpo. }\end{array}$ & $\begin{array}{l}\text { La voz, sin } \\
\text { palabras. }\end{array}$ & $\begin{array}{l}\text { Cualquier } \\
\text { objeto del } \\
\text { almacén. }\end{array}$ \\
\hline $\begin{array}{l}\text { Selección del } \\
\text { material }\end{array}$ & Profesor. & Profesor. & Profesor. & Alumnos. & Profesor. & Profesor. & Alumnos. \\
\hline $\begin{array}{l}\text { Juego des- } \\
\text { arrollado }\end{array}$ & $\begin{array}{l}\text { Habitual } \\
\text { (p.e., futbol; } \\
\text { balón volea; } \\
\text { lanzamientos } \\
\text { de pelotas). }\end{array}$ & $\begin{array}{l}\text { Habitual } \\
\text { (p.e., rotar } \\
\text { los aros). } \\
\text { Creativo } \\
\text { (p.e., punter- } \\
\text { ía; laberintos, } \\
\text { lanzamien- } \\
\text { tos). }\end{array}$ & $\begin{array}{l}\text { Habitual } \\
\text { (p.e., comba; } \\
\text { disfraces). } \\
\text { Creativo } \\
\text { (p.e., escultu- } \\
\text { ras y cua- } \\
\text { dros). }\end{array}$ & $\begin{array}{l}\text { Habitual } \\
\text { (p.e., juegos } \\
\text { de pelotas). } \\
\text { Creativo } \\
\text { (p.e. bailes, } \\
\text { construccio- } \\
\text { nes y compe- } \\
\text { ticiones } \\
\text { novedosas). }\end{array}$ & $\begin{array}{l}\text { Habitual } \\
\text { (p.e., escon- } \\
\text { dite). } \\
\text { Creativo } \\
\text { (p.e., dicta- } \\
\text { dos musica- } \\
\text { les con puer- } \\
\text { tas de arma- } \\
\text { rios). }\end{array}$ & $\begin{array}{l}\text { Habitual } \\
\text { (p.e., sonidos } \\
\text { de animales; } \\
\text { melodías). } \\
\text { Creativo } \\
\text { (p.e. piezas } \\
\text { musicales } \\
\text { integrando } \\
\text { aportaciones } \\
\text { individua- } \\
\text { les). }\end{array}$ & $\begin{array}{l}\text { Creativo } \\
\text { generalizado } \\
\text { y colectivo } \\
\text { (p.e., carreras } \\
\text { con carritos } \\
\text { inventados). } \\
\text { Participa casi } \\
\text { todo el gru- } \\
\text { po. }\end{array}$ \\
\hline $\begin{array}{l}\text { Recordar } \\
\text { norma: } \\
\text { "prohibido } \\
\text { el uso del } \\
\text { lenguaje } \\
\text { oral" }\end{array}$ & $\begin{array}{l}\text { Varias veces } \\
\text { (con seña). }\end{array}$ & $\begin{array}{l}\text { Una vez } \\
\text { (con seña). }\end{array}$ & $\begin{array}{l}\text { Se marcan } \\
\text { los que } \\
\text { hablan con } \\
\text { una tarjeta. }\end{array}$ & Ninguna. & Ninguna. & Ninguna. & Ninguna. \\
\hline $\begin{array}{l}\text { Conducta } \\
\text { final }\end{array}$ & $\begin{array}{l}\text { Uso genera- } \\
\text { lizado del } \\
\text { lenguaje oral. } \\
\text { Juegos habi- } \\
\text { tuales. } \\
\text { Búsqueda de } \\
\text { objetos nue- } \\
\text { vos. }\end{array}$ & $\begin{array}{l}\text { 1/3 del gru- } \\
\text { po: órdenes } \\
\text { orales y } \\
\text { juego habi- } \\
\text { tual. } \\
2 / 3 \text { del gru- } \\
\text { po: su juego } \\
\text { empieza a ser } \\
\text { "novedoso". }\end{array}$ & $\begin{array}{l}1 / 3 \text { del gru- } \\
\text { po: su juego } \\
\text { no evolucio- } \\
\text { na. } \\
2 / 3 \text { del gru- } \\
\text { po: realizan } \\
\text { juegos crea- } \\
\text { tivos varia- } \\
\text { dos. }\end{array}$ & $\begin{array}{l}- \text {-Se forman } \\
\text { grupos más } \\
\text { numerosos, } \\
\text { activos y } \\
\text { cómplices. } \\
\text {-Juegos más } \\
\text { fluidos, va- } \\
\text { riados y } \\
\text { elaborados. }\end{array}$ & $\begin{array}{l}\text { Gran activi- } \\
\text { dad colecti- } \\
\text { va. Juegos: } \\
\text { fluidos, } \\
\text { flexibles } \\
\text { novedosos y } \\
\text { elaborados. }\end{array}$ & $\begin{array}{l}\text { Menos canti- } \\
\text { dad de jue- } \\
\text { gos creati- } \\
\text { vos, pero } \\
\text { flexibles, } \\
\text { duraderos y } \\
\text { más elabora- } \\
\text { dos". }\end{array}$ & $\begin{array}{l}\text { Identifica- } \\
\text { ción del } \\
\text { maestro que } \\
\text { apoya y el } \\
\text { que inhibe. } \\
\text { Feedback } \\
\text { colectivo. } \\
\text { Entrenamien- } \\
\text { to general en } \\
\text { "escucha } \\
\text { empática no } \\
\text { participati- } \\
\text { va". }\end{array}$ \\
\hline $\begin{array}{l}\text { Duración } \\
\text { total sesión }\end{array}$ & $45 \mathrm{~min}$. & $60 \mathrm{~min}$. & $90 \mathrm{~min}$. & $90 \mathrm{~min}$. & $90 \mathrm{~min}$. & $90 \mathrm{~min}$. & 90 min. \\
\hline $\begin{array}{l}\text { Verbaliza- } \\
\text { ciones }\end{array}$ & $\begin{array}{l}\text { Sin relevan- } \\
\text { cia. }\end{array}$ & $\begin{array}{l}\text { Primeras } \\
\text { impresiones } \\
\text { sobre viven- } \\
\text { cias persona- } \\
\text { les, en juego } \\
\text { libre. }\end{array}$ & $\begin{array}{l}\text { Análisis de } \\
\text { vivencias y } \\
\text { del objetivo } \\
\text { que cumplían } \\
\text { las tarjetas. }\end{array}$ & $\begin{array}{l}\text { Consenso. El } \\
\text { el juego ha } \\
\text { cambiado a } \\
\text { más rápido y } \\
\text { creativo. } \\
\text { Primeras } \\
\text { creencias } \\
\text { falsas explí- } \\
\text { citas. }\end{array}$ & $\begin{array}{l}\text { Consenso. } \\
\text { Reconoci- } \\
\text { miento de } \\
\text { que ha sido } \\
\text { la sesión con } \\
\text { más creativi- } \\
\text { dad. Cambio } \\
\text { de creencias } \\
\text { anteriores. }\end{array}$ & $\begin{array}{l}\text { Análisis de } \\
\text { vivencias y } \\
\text { de emocio- } \\
\text { nes positivas } \\
\text { (asombro, } \\
\text { júbilo...). }\end{array}$ & $\begin{array}{l}\text { Identifica- } \\
\text { ción de cre- } \\
\text { encias falsas } \\
\text { en relación } \\
\text { con el rol del } \\
\text { maestro. }\end{array}$ \\
\hline
\end{tabular}

